\title{
Better living through peptide-conjugated chemistry: next-generation antisense oligonucleotides
}

\author{
Elizabeth M. McNally and Brian D. Leverson \\ Center for Genetic Medicine, Northwestern University Feinberg School of Medicine, Chicago, Illinois, USA.
}

\begin{abstract}
Two different antisense oligonucleotide-based (ASO-based) therapies are currently in clinical use to treat neuromuscular diseases. This success, for Duchenne muscular dystrophy and spinal muscular atrophy, offers hope not only for additional neuromuscular diseases, but also for other disorders that could benefit from RNA-targeted therapies. A major limitation for more widespread application of ASOs relates to relatively poor tissue penetration. In this issue of the $J C I$, Klein et al. showed that conjugating an ASO with an arginine-rich cell-penetrating peptide, Pip6a, enhanced delivery, resulting in corrective outcome for a mouse model of myotonic dystrophy. Linking ASOs to cell-penetrating peptides, or even other moieties, is an approach currently under development with treatment potential to expand to other disorders.
\end{abstract}

\section{Molecular mechanisms of myotonic dystrophy}

Myotonic dystrophy type 1 (DM1) is one of the most common neuromuscular disorders, with an estimated incidence of 1 per 8,000 in some populations. DM1 is caused by heterozygous expansion of the CTG trinucleotide repeat in the DMPK gene, and longer repeat expansions generally correlate with more severe symptoms. Furthermore, the CTG repeat expansion may be unstable in somatic tissues, and further expansion of the CTG repeat across generations leads to earlier onset and more severe symptoms. DM1 is a systemic disorder, but muscle weakness and muscle myotonia, characterized as an inability to relax contracted muscle, are among its most disabling symptoms.

The transcription of CTG repeats into RNA-based CUG sequences leads to RNA aggregates (referred to as foci), resulting in intranuclear clusters that are readily detectable using probes for CUG repeats. In DM1 cells, RNA foci colocalize with splicing regulatory factors. When RNA foci sequester muscleblind-like splicing regulator (MBNL), which is normally diffusely distributed, missplicing events ensue. Aberrant splicing in DM1 has been described in a number of key target genes, including the major muscle chloride channel, CLCN1, which is thought to underlie the symptoms of myotonia. Missplicing of other genes, for example INSR, encoding the insulin receptor, and $S C N 5 A$, which specifies the cardiac sodium channel, are thought to underlie other system disorders, such as metabolic and cardiac conduction defects. In DM1, many of the missplicing events result in expression of splice forms normally found in developing tissues. It is possible to design antisense oligonucleotides (ASOs) to target each of these missplicing events, but given the large number of misspliced mRNAs in DM1, this approach would likely fail

Related Article: p. 4739

Conflict of interest: EMM is a coauthor on patents for multiexon skipping (US Patents 9777271 and 10273483). EMM founded Ikaika Therapeutics, received grant support from Solid Biosciences, and consulted for Exonics, Invitae, AstraZeneca, Tenaya Therapeutics, 4D Molecular Therapeutics, and Cytokinetics.

Copyright: @ 2019, American Society for Clinical Investigation.

Reference information: / Clin Invest. 2019;129(11):4570-4571. https://doi.org/10.1172/JCI131933.

to address the many symptoms. An alternative approach targets the expressed CUG repeat itself, with the goal of destabilizing intranuclear foci and/or liberating the sequestered splicing factors like MBNL $(1,2)$.

\section{Clinical benefits of ASO treatment}

A safety and tolerability study of ASO treatment in DM1 patients completed enrollment (NCT0231201), but has been described as having limited clinical benefit related to poor tissue uptake of the ASOs. A number of alternative modifications are emerging that aim for better tissue delivery by taking advantage of cell-penetrating peptides. In this issue, Klein et al. describe the use and efficacy of Pip6a, a novel cell-penetrating peptide, conjugated to CUG-targeted ASOs (3). The authors evaluated the effects of Pip6a-ASOs in both a mouse model and human DM1 cells. The mouse model, HSA-LR, expresses 250 copies of the CTG repeat in skeletal muscle only under the control of the human skeletal muscle actin promoter. This model reasonably replicates the myotonia features of DM1, and after Klein and colleagues treated the mice with ASOs, key splicing events were restored. Notably, the researchers used five- to ten-fold lower cumulative doses than previously reported for other ASO-mediated treatments in this same model. Klein et al. took a comprehensive view of splicing using deep, paired-end RNA sequencing, showing that $70 \%-$ $80 \%$ of splicing defects were corrected toward normal splicing. Further, Klein et al. observed a reduction in RNA foci per nuclei as well as a reduction of the CUG-containing RNA. Like other ASO chemistries, a long-lasting and durable effect was observed, with correction of these missplicing events even six months after dosing the Pip6a-ASOs, a result thought to relate to nuclease resistance of ASOs (2). 
Klein and colleagues complemented these mouse studies by also treating myogenic cells isolated from DM1 patients, again demonstrating that Pip6a-ASOs corrected missplicing events. The human DM1 cells used in these studies displayed markedly expanded CTG repeat units (2600 and 1300 repeats), values much larger than is typically seen in adults with DM1, in which repeat expansions as low as 75-150 can be associated with significant symptoms of myotonia, progressive muscle weakness, and cardiac complications. Remarkably, treatment of the DM1 mutant cells with Pip6a-ASOs corrected splicing and reduced nuclear MBNL and RNA foci, all considered molecular signatures of correction (3). Whether Pip6a-ASOs will have improved tissue penetration into intact human muscle, heart, and brain remains to be seen, but the molecular findings are encouraging.

ASOs can target splicing regulatory sites to induce exon inclusion or exclusion. In the case of DM1, directing ASOs against a specific mutation can reduce undesirable expression and negative molecular consequences. Early generations of ASOs were typically minimally modified at the phosphodiester backbone, and these agents largely failed because of rapid degradation by nucleases (4). Other modifications that focused on the deoxyribose sugar moiety helped stabilize ASOs, but also were more likely to have certain dose-limiting toxicities. One newer-generation ASO that utilized a neutral-charge chemistry, phosphorodiamidate morpholinos (PMOs), replaced the ribose and its phosphodiester bonds with a morpholine ring forming the phosphorodiamidate linkage (5). The drug eteplirsen has successfully employed this PMO chemistry to treat Duchenne muscular dystrophy (DMD) $(6,7)$. Additionally, modifying PMOs using covalently conjugated cell-penetrating peptides or $\mathrm{PMO}$ internalization peptides (Pips) further improves cellular uptake (8). These modifications typically rely on a series of arginine residues complexed with additional sequences or compounds to enable better cell entry. The Pip6a modification (Ac-RXRRBRRXRYQFLIRXRBRXRB-OH, with B $=\beta$-alanine and $X=$ amino hexanoic acid) showed better entry into dystrophin-deficient heart and muscle cells (9).

ASOs are currently used to treat DMD, a disorder characterized as having a fragile sarcolemma with enhanced permeability. This feature of DMD skeletal muscle and cardiomyocytes is thought to enhance uptake of ASOs, even in the absence of extensive moieties that promote cell penetration. In contrast, the sarcolemma of myotonic myofibers is less permeable. Thus, advances that improve tissue penetration should translate to lower dosing, improved efficacy, and potentially, a reduced side-effect profile. Such improvement could also facilitate exon skipping and even multiple exon skipping, in which more than one exon is targeted to restore the reading frame. In addition to cell-penetrating peptides, fatty acids are being used as alternative conjugates, and early data suggest such avenues also promote tissue uptake (10). Like most first-in-class compounds, ASO conjugate chemistry is expected to progress and with greater innovation could increase tissue penetration and potentially even tissue-specific delivery methods.

\section{Acknowledgments}

EMM and BDL are supported by NIH grants HL128075, AR052646, and HL061322.

Address correspondence to: Elizabeth $\mathrm{M}$ McNally, Center for Genetic Medicine, Northwestern University Feinberg School of Medicine, Chicago, Illinois 60611, USA. Phone: 312.503.5600; Email: elizabeth. mcnally@northwestern.edu.

1. González-Barriga A, et al. Design and analysis of effects of triplet repeat oligonucleotides in cell models for myotonic dystrophy. Mol Ther Nucleic Acids. 2013;2:e81.

2. Wheeler TM, et al. Targeting nuclear RNA for in vivo correction of myotonic dystrophy. Nature. 2012;488(7409):111-115.

3. Klein AF, et al. Peptide-conjugated oligonucleotides evoke long-lasting myotonic dystrophy correction in patient-derived cells and mice. J Clin Invest. 2019;129(11):4739-4744.

4. Tsoumpra MK, Fukumoto S, Matsumoto T, Takeda S, Wood MJA, Aoki Y. Peptide-conjugate antisense based splice-correction for Duchenne muscular dystrophy and other neuromuscular diseases. EBioMedicine. 2019;45:630-645.

5. McClorey G, Banerjee S. Cell-penetrating peptides to enhance delivery of oligonucleotide-based therapeutics. Biomedicines. 2018;6(2):E51.

6. Betts CA, Hammond SM, Yin HF, Wood MJ. Optimizing tissue-specific antisense oligonucleotide-peptide conjugates. Methods Mol Biol. 2012;867:415-435

7. Aartsma-Rus A, et al. Development of exon skipping therapies for Duchenne muscular dystrophy: a critical review and a perspective on the outstanding issues. Nucleic Acid Ther. 2017;27(5):251-259.

8. Lehto T, Ezzat K, Wood MJA, El Andaloussi S. Peptides for nucleic acid delivery. Adv Drug Deliv Rev. 2016;106(Pt A):172-182.

9. Lehto $\mathrm{T}$, et al. Cellular trafficking determines the exon skipping activity of Pip6a-PMO in mdx skeletal and cardiac muscle cells. Nucleic Acids Res. 2014;42(5):3207-3217.

10. Prakash TP, et al. Fatty acid conjugation enhances potency of antisense oligonucleotides in muscle. Nucleic Acids Res. 2019;47(12):6029-6044. 